\title{
Supernetwork Representation Formulation of a Multiclass Simultaneous Transportation Equilibrium Model as a Fixed Demand User Equilibrium Problem
}

\author{
Mohamad K. Hasan, Kuwait University, Kuwait \\ iD https://orcid.org/0000-0003-1185-6034 \\ Mohammad Saoud, Kuwait University, Kuwait \\ Raed Al-Husain, Kuwait University, Kuwait
}

\begin{abstract}
A multiclass simultaneous transportation equilibrium model (MSTEM) explicitly distinguishes between different user classes in terms of socioeconomic attributes, trip purpose, pure and combined transportation modes, as well as departure time, all interacting over a physically unique multimodal network. It enhances the prediction process behaviorally by combining the trip generation and departure time choices to trip distribution, modal split, and trip assignment choices in a unified and flexible framework that has many advantages from both supply and demand sides. However, the development of this concept of multiple classes increases the mathematical complexity of travel forecasting models. In this research, the authors reduce this mathematical complexity by using the supernetwork representation formulation of the diagonalized MSTEM as a fixed demand user equilibrium (FDUE) problem.
\end{abstract}

\section{KEYWORDS}

Equivalent Optimization Program, Fixed Demand User Equilibrium, Multiclass Simultaneous (Combined) Transportation Equilibrium Model, Relaxation (Diagonalization) Algorithm, Variational Inequality

\section{INTRODUCTION}

Transportation planning models have evolved through different approaches that can be classified as follows:

- Sequential Approach: This approach has been applied to hundreds of transportation studies throughout the world for 60 years. It views transportation planning as a sequential process, often with four stages: trip generation, trip distribution, modal split, and trip assignment (Hasan \& Al-Qaheri, 2013).

This sequential approach has several inherent weakness (Tatineni et al., 1994). 
- Simultaneous (Combined) Approach, Single-Class Models: In order to overcome these deficiencies, researchers attempt to predict all four steps simultaneously (Beckmann et al., 1956). Boyce (2014) summarized Beckmann's contributions to regional science in the 1950s. Boyce and Nagurney (2019) gave a brief summary of Beckmann's seminal contributions to transportation science and location theory, which continued to stimulate this field for well over half a century. This evidences the achievement of his book as well as his other scientific publications.

Evans (1976) examined how to combine trip distribution and traffic assignment models in a single formulation. She succeeded in formulating one version of the combined model as an optimization problem with a convex objective function consisting of two parts, namely one related to route choice, similar to the objective function in Beckmann's formulation, and the other related to travel demand or trip distribution.

Florian and Nguyen (1978) extended the formulation to include a modal split. They formulated a combined trip distribution, modal split, and trip assignment model, considering two independent modes (i.e., car and transit).

Safwat and Magnanti (1988) further enriched the behavioral features of the equivalent optimization approach to include trip generation. In their model (i.e., the simultaneous transportation equilibrium model [STEM]) trip generation can depend upon the performance of the system through an accessibility measure that is based on the random utility theory of users' behavior (instead of being fixed), and trip distribution is given by a more flexible logit model based on the random utility theory (instead of being provided by a less flexible entropy model.

Hasan and Al-Gadhi, (1998) applied the STEM to the urban transportation networks of Riyadh, Saudi Arabia.

Each of the above-proposed models concerned a single-class model, in the sense that all travelers by purpose or socioeconomic group are represented as one homogeneous group.

- Simultaneous (Combined) Approach, Multiclass Models: As the authors mentioned above, simple travel forecasting models assume that all travelers are similar in their travel-decision characteristics, such as their money-value of the time and their sensitivity to travel times in choosing their origin, destination, and mode of travel. In order to obtain more realistic models, travelers are often divided into classes, either by socioeconomic attributes or by the purpose of their trip (e.g., work or shopping), assuming that travel-decision characteristics are the same within each class, but differ among classes.

The introduction of multiple classes increases the mathematical complexity of travel forecasting models (Boyce \& Bar-Gera, 2004).

De Cea et al. (2003) implemented multiclass combined models emphasizing route choices in a congested transit network with several combinations of transit modes, as they found in Santiago. This research led to the development of the computer package ESTRAUS and related software, which has been extensively applied to Santiago as well as other Chilean cities.

Yao et al. (2014) proposed an alternative formulation for the combined distribution and assignment problem, which seeks to determine consistent level-of-service.

Zhao et al. (2018) proposed a bilevel optimization model for multiclass origin-destination estimation using various types of data. The multiclass character of the model, a new feature, and significant contribution to the literature are essential because of the increasing interest in simultaneous estimation of origin-destination tables for various classes of trucks and automobiles. All the multiclass combined models the authors reviewed above, except the STEM, consider that the total originating and terminating flows are known, that is, the trip generation step of the transportation planning process is exogenous to the combined prediction process. This encourages the development of the STEM to be a multiple-user classes model in terms of the socioeconomic group (e.g., income level and car availability), trip purpose, as well as pure and combined transportation modes, interacting over a physically unique network. The developed multiclass simultaneous transportation equilibrium model (MSTEM) (Hasan \& Dashti 2007) 
also combines the departure time explicitly as one of the main components of the prediction process for the first time.

The developed MSTEM can be considered the state of the art of the multiclass combined models that include the most recent features of other multiclass combined models, in addition to new additional features.

However, the development of this concept of multiple classes increases the mathematical complexity of travel forecasting models. In this paper, the authors try to reduce this mathematical complexity by using the supernetwork representation formulation of the MSTEM as a fixed demand user equilibrium (FDUE) problem.

This paper is organized as follows: The following section illustrates the MSTEM modeling procedure; the third section provides a detailed formulation of the equivalent convex program (ECP) of the MSTEM as a FDUE problem that can be solved by a greedy path-based algorithm for traffic assignment (Xie et al. 2018) and offer a proof that this FDUE is equivalent to the diagonalized MSTEM; the fourth section presents future research directions; finally, the fifth sectionsummarizes the study and provides conclusions.

\section{THE MULTICLASS SIMULTANEOUS TRANSPORTATION EQUILIBRIUM MODEL}

In this section, the authors give a brief description of the MSTEM Hasan and Dashti (2007) developed in their work.

\section{Notation}

Let $(N, A)$ be a multimodal network consisting of a set of $N$ nodes and a set of $A$ links that can represent any mode of transport $m$ in an urban area. These modes can be grouped into different nests $n$ that could be multiple pure and combined (combination of pure) modes. A typical user of class $l$ with trip purpose $o$ traveling from a given origin $i$ at a specific departure time $t$ to any destination $j$ that is accessible from $i$ can use any of these modes for his/her journey. The authors will use the following notation for the MSTEM modeling:

$(N, A)=$ A multimodal network consisting of a set of $N$ nodes and a set of $A$ links.

$l=$ User class (e.g., income level and car availability).

$L=$ Set of all user classes.

$o=$ Trip purpose (e.g., home-based-work and home-based-shopping).

$O=$ Set of all trip purpose.

$I^{l o}=$ Set of origin nodes for user class $l$ and trip purpose $o$.

$i=$ An origin node in the set $I^{l o}$ for user class $l$ with the trip purpose $o$.

$D_{i}^{l o}=$ Set of destination nodes that are accessible from a given origin $i$ for the user class $l$ with trip purpose $o$.

$j=$ A destination node in the set $D_{i}^{l o}$ for user class $l$ with the trip purpose $o$.

$R^{l o}=$ Set of origin-destination pairs $i j$ for user class $l$ with trip purpose $o$, that is, the set of all origins $i \in I^{l o}$ and destinations $j \in D_{i}^{l o}$.

$m=$ Any transportation mode in the urban area.

$n=$ Nest of transportation modes $m$ that has a specific characteristic (e.g., pure modes including private and public or combined modes) that are available for user class $l$ with trip purpose $o$ travel between origin-destination pairs $i j$.

$\Lambda_{i j}^{l o}=$ Set of all nests of modes $n$ that are available for user class $l$ with the trip purpose $o$ travel between origin-destination pairs $i j$. 
$M_{n}^{l o}=$ Set of all transportation modes $m$ in the nest $n$ for user class $l$ with a trip purpose $o$ travel between origin-destination pairs $i j$.

$t=$ Departure time for user class $l$ with trip purpose $o$ using mode $m$ in the nest $n$ to travel between origin-destination pairs $i j$.

$K_{m}^{l o}=$ Time horizon of the departure periods $t$ for users of class $l$ with a trip purpose $o$ using mode $m$ between origin-destination pairs $i j$.

$p=$ A simple (i.e., no node repeated) multimodal path (i.e., it may include links with combined modes $m$ ) in the multimodal network $(N, A)$.

$P_{i j}^{\text {lonmt }}=$ Set of simple paths for travel from the origin node $i$ to the destination node $j$ in the multimodal network $(N, A)$ for users of class $l$ with trip purpose $o$ departs at a time $t \in K_{m}^{l o}$ using mode $m \in M_{n}^{l o}$ from the nest of modes $n \in \Lambda_{i j}^{l o}$.

$a=\mathrm{A}$ link in the set $A$ in the multimodal network $(N, A)$.

$u_{i j}^{\text {lonmt }}=$ The perceived minimum (generalized) cost of travel for users of class $l$ with trip purpose $o$ depart at a time $t$ using mode $m$ in the nest $n$ from the origin node $i$ to destination node $j$ in the set $D_{i}^{l o}$.

$A_{j}^{l o}=$ A composite measure of the effect that socio-economic variables, which are exogenous to the transport system and have on the trip attraction at the destination $j$ for users of class $l$ with trip purpose $o$.

$S_{i}^{l o}=$ The accessibility of origin $i \in I^{l o}$ as perceived by a user of class $l$ with the trip purpose $o$ traveling from that origin.

$G_{i}^{l o}=$ The number of trips generated from the origin $i$ for users of class $l$ with trip purpose $o$.

$E_{i}^{l o}=$ A composite measure of the effect of the socioeconomic variables, which are exogenous to the transport system and have on the number of trips generated from the origin $i$ for users of class $l$ with trip purpose.

$T_{i j}^{l o}$ Number of trips of users of class $l$ with trip purpose $o$ traveling from the origin node $i \in I^{l o}$ to the destination node $j \in D_{i}^{l o}$.

$T_{i j}^{l o n}=$ Number of trips of users of class $l$ with trip purpose $o$ traveling from the origin node $i \in I^{l o}$ to the destination node $j \in D_{i}^{l o}$ and who already chose the nest of modes.

$T_{i j}^{l o n m}=$ Number of trips of users of class $l$, with trip purpose $o$ traveling from the origin node $i \in I^{l o}$ to the destination node $j \in D_{i}^{l o}$ and who already chose the mode of transport $m \in M_{n}^{l o}$ from the nest of modes $n \in \Lambda_{i j}^{l o}$.

$T_{i j}^{l o n m t}=$ Number of trips of users of class $l$, with trip purpose $o$ traveling from the origin node $i \in I^{l o}$ to the destination node $j \in D_{i}^{l o}$, who already chose the mode of transport $m \in M_{n}^{l o}$ from the nest of modes $n \in \Lambda_{i j}^{l o}$ and start their trip at the time $t \in K_{m}^{l o}$.

$C_{a}^{\text {lonmt }}=C_{a}^{\text {lonmt }}(\boldsymbol{f})=$ the link cost function, $\forall a \in A, \forall t \in K_{m}^{l o}, \forall m \in M_{n}^{l o}, \forall n \in \Lambda_{i j}^{l o}, \forall i j \in R^{l o}$, $\forall l \in L, \forall o \in O$, will depend, in general, upon the flow over all links, the vector $\mathbf{f}$, in the multimodal network $(N, A)$ for all user class $l \in L$, trip propose $o \in O$, transport mode nest $n \in \Lambda_{i j}^{l o}$, transport mode $m \in M_{n}^{l o}$, and departure time $t \in K_{m}^{l o}$,

where $\mathbf{f}=\left(f_{a}^{\text {lonmt }}: a \in A, t \in K_{m}^{l o}, m \in M_{n}^{l o}, n \in \Lambda_{i j}^{l o}, i j \in R^{l o}, l \in L, o \in O\right)$ 
The authors will also assume that the perceived cost of travel on any multimodal route (path) $p \in P_{i j}^{\text {lonmt }}$ is the sum of travel costs on the links that comprise that path, that is:

$C_{p}^{\text {lonmt }}=\sum_{a \in A} \delta_{a p}^{\text {lonmt }} C_{a}^{\text {lonmt }}(\mathbf{f}), \forall p \in P_{i j}^{l o n m t}, \forall t \in K_{m}^{l o}, \forall m \in M_{n}^{l o}, \forall n \in \Lambda_{i j}^{l o}, \forall i j \in R^{l o}, \forall l \in L, \forall o \in O$

where

$\delta_{a p}^{\text {lonmt }}=\left\{\begin{array}{ll}1 & \text { if link } a \text { belongs to path } p \\ 0 & \text { otherwise }\end{array}\right\}$

$\forall p \in P_{i j}^{l o n m t}, \forall t \in K_{m}^{l o}, \forall m \in M_{n}^{l o}, \forall n \in \Lambda_{i j}^{l o}, \forall i j \in R^{l o}, \forall l \in L, \forall o \in O$

\section{THE MULTICLASS SIMULTANEOUS TRANSPORTATION EQUILIBRIUM MODEL}

$$
S_{i}^{l o}=\max \left\{0, \ln \sum_{j \in D_{i}^{l o}} \sum_{n \in \Lambda_{i j}^{l o}} \sum_{m \in M_{n}^{l o}} \sum_{t \in K_{m}^{l o}} \exp \left(-,{ }_{i}^{l o} u_{i j}^{l o n m t}+A_{j}^{l o}\right)\right\} \quad \forall i \in I^{l o}, \forall l \in L, \forall o \in O
$$

$G_{i}^{l o}=\alpha^{l o} S_{i}^{l o}+E_{i}^{l o}$

$\forall i \in I^{l o}, \forall l \in L, \forall o \in O$

$T_{i j}^{l o}=G_{i}^{l o} \frac{\sum_{n \in \Lambda_{i j}^{l o}} \sum_{m \in M_{n}^{l o}} \sum_{t \in K_{m}^{l o}} \exp \left(-,{ }_{i}^{l o} u_{i j}^{\text {lonmt }}+A_{j}^{l o}\right)}{\sum_{j \in D_{i}^{l o}} \sum_{n \in \Lambda_{i j}^{l o}} \sum_{m \in M_{n}^{l o}} \sum_{t \in K_{m}^{l o}} \exp \left(-,{ }_{i}^{l o} u_{i j}^{l o n m t}+A_{j}^{l o}\right)} \quad \forall i j \in R^{l o}, \forall l \in L, \forall O \in O$

$T_{i j}^{l o n}=T_{i j}^{l o} \frac{\sum_{m \in M_{n}^{l o}} \sum_{t \in K_{m}^{l o}} \exp \left(-,{ }_{i}^{l o} u_{i j}^{l o n m t}+A_{j}^{l o}\right)}{\sum_{n \in \Lambda_{i j}^{l o}} \sum_{m \in M_{n}^{l o}} \sum_{t \in K_{m}^{l o}} \exp \left(-,{ }_{i}^{l o} u_{i j}^{l o n m t}+A_{j}^{l o}\right)} \quad, \forall n \in \Lambda_{i j}^{l o}, \forall i j \in R^{l o}, \forall l \in L, \forall o \in O$

$T_{i j}^{l o n m}=T_{i j}^{l o n} \frac{\sum_{t \in K_{m}^{l o}} \exp \left(-,{ }_{i}^{l o} u_{i j}^{\text {lonmt }}+A_{j}^{l o}\right)}{\sum_{m \in M_{n}^{l o}} \sum_{t \in K_{m}^{l o}} \exp \left(-,{ }_{i}^{l o} u_{i j}^{\text {lonmt }}+A_{j}^{l o}\right)}, \forall m \in M_{n}^{l o}, \forall n \in \Lambda_{i j}^{l o}, \forall i j \in R^{l o}, \forall l \in L, \forall O \in O$ 


$$
T_{i j}^{\text {lonmt }}=T_{i j}^{\text {lonm }} \frac{\exp \left(-\theta_{i}^{l o} u_{i j}^{l o n m t}+A_{j}^{l o}\right)}{\sum_{t \in K_{m}^{l o}} \exp \left(-,{ }_{i}^{l o} u_{i j}^{l o n m t}+A_{j}^{l o}\right)}, \forall t \in K_{m}^{l o}, \forall m \in M_{n}^{l o}, \forall n \in \Lambda_{i j}^{l o}, \forall i j \in R^{l o}, \forall l \in L, \forall o \in O
$$

$$
C_{p}^{\text {lonmt }}\left\{\begin{array}{lll}
=u_{i j}^{\text {lonmt }} & \text { if } & h_{p}^{\text {lonmt }}>0 \\
\geq u_{i j}^{\text {lonmt }} & \text { if } & h_{p}^{\text {lonmt }}=0
\end{array}\right\}, \forall p \in P_{i j}^{\text {lonmt }}, \forall t \in K_{m}^{l o}, \forall m \in M_{n}^{l o}, \forall n \in \Lambda_{i j}^{l o}, \forall i j \in R^{l o}, \forall l \in L, \forall o \in O
$$

where

$$
\begin{aligned}
& C_{p}^{\text {lonmt }}=\sum_{a \in A} \delta_{a p}^{\text {lonmt }} C_{a}^{\text {lonmt }}(\mathbf{f}), \forall p \in P_{i j}^{\text {lonmt }}, \forall t \in K_{m}^{l o}, \forall m \in M_{n}^{l o}, \forall n \in \Lambda_{i j}^{l o}, \forall i j \in R^{l o}, \forall l \in L, \forall o \in O \\
& \delta_{a p}^{\text {lonmt }}=\left\{\begin{array}{ll}
1 & \text { if link } a \text { belongs to path } p \\
0 & \text { otherwise }
\end{array}\right\}, \\
& \mathbf{f}=\left(f_{a}^{\text {lonmt }}: a \in A, t \in K_{m}^{l o}, m \in M_{n}^{l o}, n \in \Lambda_{i j}^{l o}, i j \in R^{l o}, l \in L, o \in O\right) \\
& f_{a}^{\text {lonmt }}=\sum_{l \in L} \sum_{o \in O} \sum_{i j \in R^{l o}} \sum_{p \in P_{i j}^{l o n m t}} \delta_{a p}^{\text {lonmt }} h_{p}^{\text {lonmt }}, \\
& \forall a \in A, \forall t \in K_{m}^{l o}, \forall m \in M_{n}^{l o}, \forall n \in \Lambda_{i j}^{l o}, \forall i j \in R^{l o}, \forall l \in L, \forall o \in O
\end{aligned}
$$

Because of the asymmetry of the link cost functions, the MSTEM cannot be cast as an equivalent optimization program as the STEM. Instead, it can be formulated as the following variational inequality (VI).

$\mathbf{C}\left(\mathbf{f}^{*}\right)^{T}\left(\mathbf{f}-\mathbf{f}^{*}\right)-\mathbf{U}\left(\mathbf{T}^{*}\right)^{T}\left(\mathbf{T}-\mathbf{T}^{*}\right) \geq \mathbf{0} \quad \forall$ feasible $\mathbf{f}, \mathbf{T}$

where

$\mathbf{f}=$ Vector of flow on links of the multimodal network.

$\mathbf{f}^{*}=$ Vector of equilibrium flow on links of the multimodal network.

$\mathbf{T}=$ Vector of trips between origin-destination pairs of the multimodal network

$\mathbf{T}=\left(\mathbf{T}^{i, l o}: i \in I^{l o}, l \in L, o \in O\right)$.

$\mathbf{T}^{*}=$ Vector of equilibrium trips between origin-destination pairs of the multimodal Network.

$\mathbf{C}\left(\mathbf{f}^{*}\right)=$ Column-vector of network link's cost functions (with nondiagonal and asymmetric Jacobian).

$\mathbf{U}\left(\mathbf{T}^{*}\right)=$ Column-vector of inverse demand functions (with nondiagonal and symmetric 
Jacobian $), \mathbf{U}=\left(\mathbf{u}^{i, l o}: i \in I^{l o}, l \in L, o \in O\right)$.

The VI problem in Equation 8 is equivalent to the MSTEM (see, for example, Dafermos, 1982, for a formal proof of equivalency between VI and traffic equilibrium) and can be solved by the relaxation (diagonalization) algorithm (Dafermos, 1982; Florian \& Spiess, 1982).

At each iteration of the diagonalization algorithm, the cost functions $C_{a}^{\text {lonmt }}$ result in the diagonalized cost function $\hat{C}_{a}^{\text {lonmt }}$, which depends only on their flows, $f_{a}^{\text {lonmt }}$, and the following VI should be solved:

$\hat{\mathbf{C}}\left(\mathbf{f}^{*}\right)^{T}\left(\mathbf{f}-\mathbf{f}^{*}\right)-\mathbf{U}\left(\mathbf{T}^{*}\right)^{T}\left(\mathbf{T}-\mathbf{T}^{*}\right) \geq \mathbf{0} \quad \forall$ feasible $\mathbf{f}, \mathbf{T}$

This VI can be formulated as ECP (Hasan \& Dashti, 2007).

\section{THE FORMULATION OF THE EQUIVALENT CONVEX PROGRAM OF THE MULTICLASS SIMULTANEOUS TRANSPORTATION EQUILIBRIUM MODEL AS A FIXED DEMAND USER EQUILIBRIUM PROBLEM}

\section{Supernetwork Representation}

In this subsection, the authors show that by a modification of the basic network, it is possible to formulate the STEM as an FDUE problem. Figure 1 shows the required adjustment; the example network (which consists of two origins and three destinations) is augmented by:

1. Dummy links leading from each destination node $j \in D_{i}^{l o}$ in the basic network to a dummy destination nodes denoted by $i^{t} \forall i \in I^{l o}, \forall t \in K_{m}^{l o}$. The flow on each of the dummy links, from $j$ to $i^{t} \forall i \in I^{l o}, \forall t \in K_{m}^{l o}$, is $T_{i j}^{\text {lonmt }}, \forall i \in I^{l o}, \forall t \in K_{m}^{l o}$, and the equivalent travel time (cost) on each of these dummy links is $\left[\ln \left(T_{i j}^{\text {lonmt }}\right)-A_{j}^{l o}\right]$.

2. Dummy links leading from each dummy destination node $i^{t} \forall i \in I^{l o}, \forall t \in K_{m}^{l o}$ in the supernetwork to dummy super destination nodes denoted by. The flow on each of these dummy links is and time (cost) is zero.

3. Dummy links leading from each origin node in the basic network $i \in I^{l o}$ to the dummy super destination nodes $i^{\prime} \forall i \in I^{l o}$. The flow on each of these dummy links is $e_{i}^{l o}=Q_{i}^{l o}-G_{i}^{l o}$, where

$Q_{i}^{l o}$ is the maximum number of trips which may be generated from the origin $i^{l o}$. The travel time (cost) on each of these dummy links is $\left[\ln \left(Q_{i}^{l o}-e_{i}^{l o}\right)-\frac{1}{\alpha^{l^{o}}}\left(Q_{i}^{l_{0}}-E_{i}^{l o}-e_{i}^{l o}\right)\right]$.

Consider now an FDUE problem defined over the modified supernetwork where the fixed demand is $Q_{i}^{l o} \forall l, o$ which must be assigned between $i \forall i \in I^{l o}$ and $i^{\prime} \forall i \in I^{l o} \forall l, o$. This problem will be formulated in the next section.

\section{Fixed Demand User Equilibrium}

The MSTEM can be formulated by the following FDUE ECP, which is needed for Step 1 of the relaxation algorithm that solves the VI formulation of the MTEM.

FDUE (ECP): 
Figure 1. Supernetwork representation for the multiclass simultaneous transportation equilibrium model

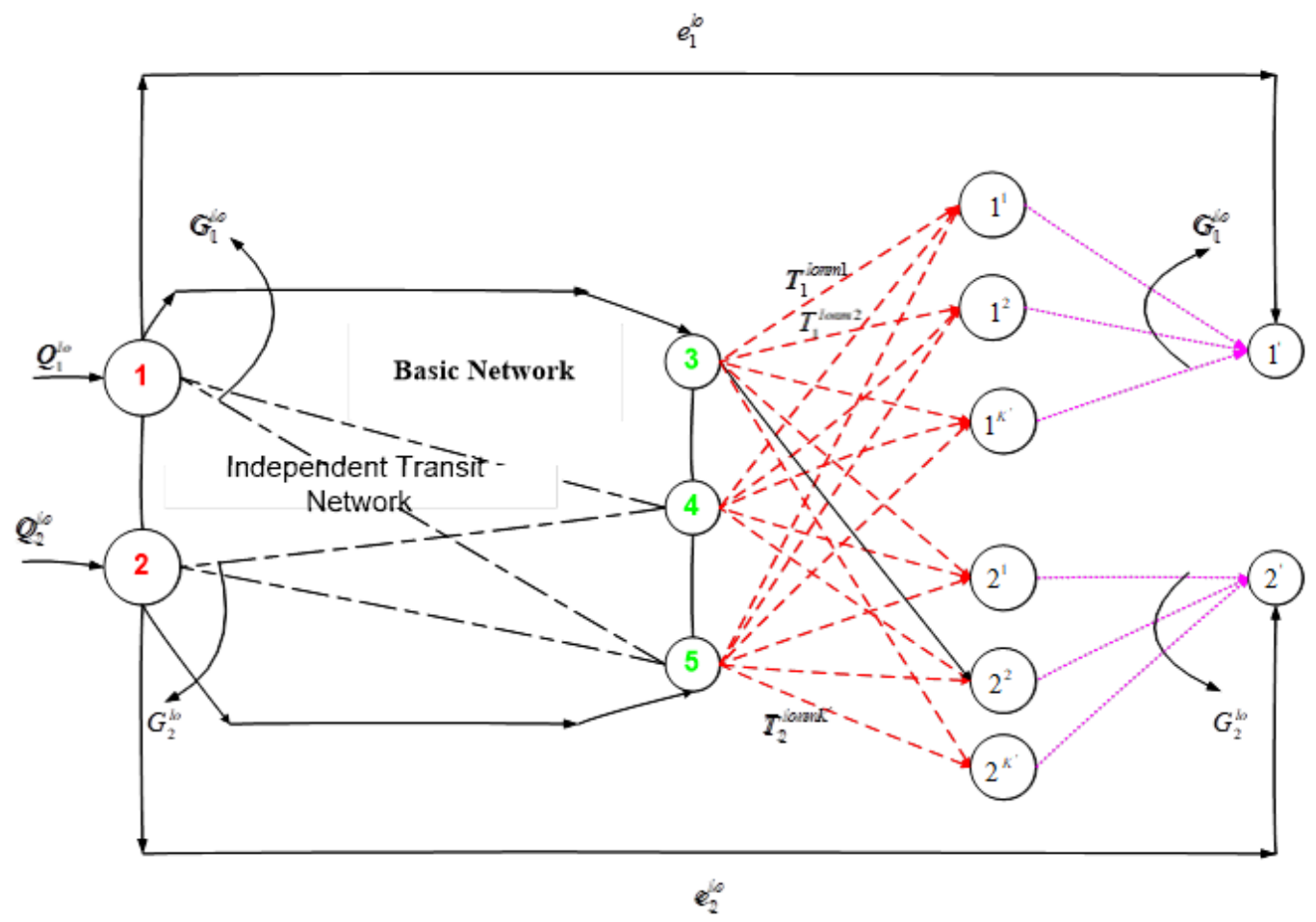

$\operatorname{Min} Z(h, T, e)=\sum_{l \in L} \sum_{o \in O} \sum_{i \in I^{l o}} \sum_{j \in D_{i}^{l o}} \sum_{n \in \Lambda_{i j}^{l o}} \sum_{m \in M_{m}^{l o}} \sum_{t \in K_{m}^{l o}} \sum_{a \in A} \int_{0}^{f_{a}^{\text {lonmt }}} \widehat{C}_{a}^{\text {lonmt }}(x) d x$

$+\sum_{l \in L} \sum_{o \in O} \sum_{i \in I^{l o}} \frac{1}{\theta_{i}^{l o}} \int_{0}^{e_{i}^{l o}}\left[\ln \left(Q_{i}^{l o}-x\right)-\frac{1}{\alpha^{l o}}\left(Q_{i}^{l o}-E_{i}^{l o}-x\right)\right] d x$

$+\sum_{l \in L} \sum_{o \in O} \sum_{i \in I^{l o}} \frac{1}{\theta_{i}^{l o}} \sum_{j \in D_{i}^{l o}} \sum_{n \in \Lambda_{i j}^{l o}} \sum_{m \in M_{n}^{l o}} \sum_{t \in K_{m}^{l o}} \int_{0}^{T_{i j}^{l o n m t}}\left[\ln (x)-A_{j}^{l o}\right] d x$

subject to:

$\Sigma_{j \in D_{i}^{l o}} T_{i j}^{l o}+e_{i}^{l o}=Q_{i}^{l o} \forall i \in I^{l o}, \forall l \in L, \forall o \in O$

$\Sigma_{n \in \Lambda_{i j}^{l o}} T_{i j}^{l o n}=T_{i j}^{l o} \forall i \in I^{l o}, \forall j \in D_{i}^{l o}, \forall l \in L, \forall o \in O$

$\Sigma_{m \in M_{n}^{l o}} T_{i j}^{l o n m}=T_{i j}^{l o n} \forall i \in I^{l o}, \forall j \in D_{i}^{l o}, \forall n \in \Lambda_{i j}^{l o}, \forall l \in L, \forall o \in O$ 


$$
\Sigma_{t \in K_{m}^{l o}} T_{i j}^{l o n m t}=T_{i j}^{l o n m} \forall i \in I^{l o}, \forall j \in D_{i}^{l o}, \forall m \in M_{n}^{l o}, \forall n \in \Lambda_{i j}^{l o}, \forall l \in L, \forall o \in O
$$

$$
\begin{aligned}
& \sum_{p \in P_{i j}^{\text {lonmt }}} h_{p}^{\text {lonmt }}=T_{i j}^{\text {lonmt }} \forall i \in I^{l o}, \forall j \in D_{i}^{l o}, \forall t \in K_{m}^{l o}, \forall m \in M_{n}^{l o}, \\
& \forall n \in \Lambda_{i j}^{l o}, \forall l \in L, \forall o \in O
\end{aligned}
$$

$S_{i}^{l o} \geq 0 \forall i \in I^{l o}, \forall l \in L, \forall o \in O$

$T_{i j}^{l o} \geq 0 \forall i \in I^{l o}, \forall j \in D_{i}^{l o}, \forall l \in L, \forall o \in O$

$T_{i j}^{l o n} \geq 0 \forall i \in I^{l o}, \forall j \in D_{i}^{l o}, \forall n \in, \forall l \in L, \forall o \in O$

$T_{i j}^{l o n m} \geq 0 \forall i \in I^{l o}, \forall j \in D_{i}^{l o}, \forall m \in, \forall n \in, \forall l \in L, \forall o \in O$

$T_{i j}^{l o n m t} \geq 0 \forall i \in I^{l o}, \forall j \in D_{i}^{l o}, \forall t \in K_{m}^{l o}, \forall m \in, \forall n \in, \forall l \in L, \forall o \in O$

$h_{p}^{\text {lonmt }} \geq 0 \quad \forall i \in I^{l o}, \forall j \in D_{i}^{l o}, \forall t \in, \forall m \in, \forall n \in, \forall l \in L, \forall o \in O$,

$p \in P_{i j}^{\text {lonmt }}$

where:

$$
\begin{aligned}
f_{a}^{\text {lonmt }}=\sum_{l \in L} \sum_{o \in O} \sum_{i j \in R^{l o}} \sum_{p \in P_{i j}^{\text {lonmt }}} \delta_{a p}^{\text {lonmt }} h_{p}^{\text {lonmt }}, \forall a \in A, \forall t \in, \forall m \in, \forall n \in, & , \forall i j \in R^{l o}, \forall l \in L, \forall o \in O
\end{aligned}
$$

This FDUE problem can be solved much easier by a greedy path-based algorithm for traffic assignment (Xie et al., 2018).

\section{Equivalency}

The ECP is equivalent to the diagonalized MSTEM (i.e., with $\mathbf{C}=\hat{\mathbf{C}}$ )

Proof

Let 
$\gamma_{i}^{l o}$ for $i \in I^{l o}, l \in L, o \in O$;

$\mu_{1, i j}^{l o}$ for $i \in I^{l o}, j \in D_{i}^{l o}, l \in L, o \in O$;

$\mu_{2, i j}^{l o n}$ for $i \in I^{l o}, j \in D_{i}^{l o}, n \in \Lambda_{i j}^{l o}, l \in L, o \in O ; \mu_{3, i j}^{l o n m}$ for $i \in I^{l o}, j \in D_{i}^{l o}, m \in, n \in, l \in L, o \in O$;

$u_{i j}^{\text {lonmt }}$ for $i \in I^{l o}, j \in D_{i}^{l o}, t \in K_{m}^{l o}, m \in M_{n}^{l o}, n \in \Lambda_{i j}^{l o}, l \in L, o \in O$;

$\eta_{i}^{l o}$ for $i \in I^{l o}, l \in L, o \in O$;

$\beta_{1, i j}^{l o}$ for $i \in I^{l o}, j \in D_{i}^{l o}, l \in L, o \in O$;

$\beta_{2, i j}^{l o n}$ for $i \in I^{l o}, j \in D_{i}^{l o}, n \in \Lambda_{i j}^{l o}, l \in L, o \in O$;

$\beta_{3, i j}^{l o n m}$ for $i \in I^{l o}, j \in D_{i}^{l o}, m \in M_{n}^{l o}, n \in \Lambda_{i j}^{l o}, l \in L, o \in O$;

$\beta_{4, i j}^{\text {lonmt }}$ for $i \in I^{l o}, j \in D_{i}^{l o}, t \in K_{m}^{l o}, m \in M_{n}^{l o}, n \in \Lambda_{i j}^{l o}, l \in L, o \in O$;

$\omega_{p}^{\text {lonmt }}$ for $p \in P_{i j}^{\text {lonmt }}, t \in K_{m}^{l o}, m \in M_{n}^{l o}, n \in \Lambda_{i j}^{l o}, i j \in R^{l o}, l \in L, o \in O$

Denote, respectively, the Karush-Kuhn-Tucker multipliers associate with constraints (9)-(13) and the six sets of nonnegativity conditions in constraints (14)-(19). Then the optimality conditions are given by the constraints in the ECP (i.e., constraints (9)-(19)) together with the following conditions:

$$
\begin{aligned}
& \frac{1}{\theta_{i}^{l o}}\left[\ln \left(Q_{i}^{l o}-e_{i}^{l o}\right)-\frac{1}{\alpha^{l o}}\left(Q_{i}^{l o}-E_{i}^{l o}-e_{i}^{l o}\right]+\gamma_{i}^{l o}-\eta_{i}^{l o}=0 \forall i \in I^{l o}, \forall l \in L, \forall o \in O\right. \\
& \gamma_{i}^{l o}+\mu_{1, i j}^{l o}-\beta_{1, i j}^{l o}=0 \forall i \in I^{l o}, \forall j \in D_{i}^{l o}, \forall l \in L, \forall o \in O \\
& -\mu_{1, i j}^{l o}+\mu_{2, i j}^{\text {lon }}-\beta_{2, i j}^{l o n}=0 \forall i \in I^{l o}, \forall j \in D_{i}^{l o}, \forall n \in \Lambda_{i j}^{l o}, \forall l \in L, \forall o \in O \\
& -\mu_{2, i j}^{\text {lon }}+\mu_{3, i j}^{\text {lonm }}-\beta_{3, i j}^{\text {lonm }}=0 \forall i \in I^{l o}, \forall j \in D_{i}^{l o}, \forall m \in M_{n}^{l o}, \forall n \in \Lambda_{i j}^{l o}, \forall l \in L, \forall o \in O \\
& \frac{1}{\theta_{i}^{l o}}\left[\ln T_{i j}^{\text {lonmt }}-A_{j}^{l o}\right]-\mu_{3, i j}^{\text {lonm }}+u_{i j}^{\text {lonmt }}-\beta_{4, i j}^{\text {lonmt }}=0 \\
& \forall i \in I^{l o}, \forall j \in D_{i}^{l o}, \forall t \in K_{m}^{l o}, \forall m \in M_{n}^{l o}, \forall n \in \Lambda_{i j}^{l o}, \forall l \in L, \forall o \in O \\
& \sum_{a \in A} \delta_{a p}^{\text {lonmt }} \hat{C}_{a}^{\text {lonmt }}\left(f_{a}^{\text {lonmt }}\right)-u_{i j}^{\text {lonmt }}-\omega_{p}^{\text {lonmt }}=0
\end{aligned}
$$


$\forall i \in I^{l o}, \forall j \in D_{i}^{l o}, \forall t \in K_{m}^{l o}, \forall m \in M_{n}^{l o}, \forall n \in \Lambda_{i j}^{l o}, \forall l \in L, \forall o \in O, p \in P_{i j}^{l o n m t}$

$S_{i}^{l o} \eta_{i}^{l o}=0$ and $\eta_{i}^{l_{0}} \geq 0 \quad \forall i \in I^{l o}, \forall l \in L, \forall o \in O$

$T_{i j}^{l o} \beta_{1, i j}^{l o}=0$ and $\beta_{1, i j}^{l o} \geq 0 \forall i \in I^{l o}, \forall j \in D_{i}^{l o}, \forall l \in L, \forall o \in O$

$T_{i j}^{l o n} \beta_{2, i j}^{l o n}=0$ and $\beta_{2, i j}^{l o n} \geq 0 \forall i \in I^{l o}, \forall j \in D_{i}^{l o}, \forall n \in \Lambda_{i j}^{l o}, \forall l \in L, \forall o \in O$

$T_{i j}^{l o n m} \beta_{3, i j}^{l o n m}=0$ and $\beta_{3, i j}^{l o n m} \geq 0 \forall i \in I^{l o}, \forall j \in D_{i}^{l o}, \forall m \in M_{n}^{l o}, \forall n \in \Lambda_{i j}^{l o}, \forall l \in L, \forall o \in O$

$T_{i j}^{\text {lonmt }} \beta_{4, i j}^{\text {lonmt }}=0$ and $\beta_{4, i j}^{\text {lonmt }} \geq 0$

$\forall i \in I^{l o}, \forall j \in D_{i}^{l o}, \forall t \in K_{m}^{l o}, \forall m \in M_{n}^{l o}, \forall n \in \Lambda_{i j}^{l o}, \forall l \in L, \forall o \in O$

$h_{p}^{\text {lonmt }} \omega_{p}^{\text {lonmt }}=0$ and $\omega_{p}^{\text {lonmt }} \geq 0$

$\forall i \in I^{l o}, \forall j \in D_{i}^{l o}, \forall t \in K_{m}^{l o}, \forall m \in M_{n}^{l o}, \forall n \in \Lambda_{i j}^{l o}, \forall l \in L, \forall o \in O, p \in P_{i j}^{l o n m t}$

At equilibrium, since $T_{i j}^{l o}, T_{i j}^{\text {lon }}, T_{i j}^{\text {lonm }}, T_{i j}^{\text {lonmt }} \succ 0$, then from Equations 27, 28, 29, and 30 $\beta_{1, i j}^{l o}=0, \beta_{2, i j}^{\text {lon }}=0, \beta_{3, i j}^{\text {lonm }}=0$, and $\beta_{4, i j}^{\text {lonmt }}=0$. Substituting these results in Equations 21, 22, 23 , and 24 and adding the resultant equations, the outcome gives:

$\frac{1}{\theta_{i}^{l o}}\left[\ln T_{i j}^{\text {lonmt }}-A_{j}^{l o}\right]+\gamma_{i}^{l o}+u_{i j}^{\text {lonmt }}=0$

$\ln T_{i j}^{\text {lonmt }}=-\theta_{i}^{l o} \gamma_{i}^{l o}-\theta_{i}^{l o} u_{i j}^{\text {lonmt }}+A_{j}^{l o}$

$T_{i j}^{l o n m t}=\exp \left(-\theta_{i}^{l o} \gamma_{i}^{l o}\right) \cdot \exp \left(-\theta_{i}^{l o} u_{i j}^{\text {lonmt }}+A_{j}^{l o}\right)$

Summing Equation 32 over all $t \in K_{m}^{l o}$ and using constraint (12) of the ECP, the result is:

$T_{i j}^{l o n m}=\exp \left(-\theta_{i}^{l o} \gamma_{i}^{l o}\right) \cdot \sum_{t \in K_{m}^{l o}} \exp \left(-\theta_{i}^{l o} u_{i j}^{l o n m t}+A_{j}^{l o}\right)$

Dividing Equation 32 by Equation 33 gives: 
$T_{i j}^{\text {lonmt }}=T_{i j}^{\text {lonm }} \frac{\exp \left(-\theta_{i}^{l o} u_{i j}^{\text {lonmt }}+A_{j}^{\text {lo }}\right)}{\sum_{t \in K_{m}^{l o}} \exp \left(-,{ }_{i}^{l o} u_{i j}^{\text {lonmt }}+A_{j}^{l o}\right)}$

which is Equation 6 in the MSTEM.

Summing Equation 33 over all $m \in M_{n}^{l o}$ and using constraint (11) of the ECP gives:

$$
T_{i j}^{l o n}=\exp \left(-\theta_{i}^{l o} \gamma_{i}^{l o}\right) \cdot \sum_{m \in M_{n}^{l o}} \sum_{t \in K_{m}^{l o}} \exp \left(-\theta_{i}^{l o} u_{i j}^{l o n m t}+A_{j}^{l o}\right)
$$

Dividing Equation 33 by Equation 34 gives:

$$
T_{i j}^{l o n m}=T_{i j}^{l o n} \frac{\sum_{t \in K_{m}^{l o}} \exp \left(-,{ }_{i}{ }^{l o} u_{i j}^{\text {lonmt }}+A_{j}^{l o}\right)}{\sum_{m \in M_{n}^{l o}} \sum_{t \in K_{m}^{l o}} \exp \left(-,{ }_{i}{ }_{i}^{l o} u_{i j}^{\text {lonmt }}+A_{j}^{l o}\right)}
$$

which is Equation (5) in the MSTEM.

Summing Equation 34 over all $n \in \Lambda_{i j}^{l o}$ and using constraint (10) of the ECP gives:

$$
T_{i j}^{l o}=\exp \left(-\theta_{i}^{l o} \gamma_{i}^{l o}\right) \cdot \sum_{n \in \Lambda_{i j}^{l o}} \sum_{m \in M_{n}^{l o}} \sum_{t \in K_{m}^{l o}} \exp \left(-\theta_{i}^{l o} u_{i j}^{l o n m t}+A_{j}^{l o}\right)
$$

Dividing Equation 34 by Equation 35 gives:

$$
T_{i j}^{l o n}=T_{i j}^{l o} \frac{\sum_{m \in M_{n}^{l o}} \sum_{t \in K_{m}^{l o}} \exp \left(-,{ }_{i}^{l o} u_{i j}^{\text {lonmt }}+A_{j}^{l o}\right)}{\sum_{n \in \Lambda_{i j}^{l o}} \sum_{m \in M_{n}^{l o}} \sum_{t \in K_{m}^{l o}} \exp \left(-,{ }_{i}^{l o} u_{i j}^{\text {lonmt }}+A_{j}^{l o}\right)}
$$

which is Equation (24) in the MSTEM.

Summing Equation 35 over all $j \in D_{i}^{l o}$ and using constraint (9) of the ECP gives:

$$
\alpha^{l o} S_{i}^{l o}+E_{i}^{l o}=G_{i}^{l o}=\exp \left(-\theta_{i}^{l o} Y_{i}^{l o}\right) \sum_{j \in D_{i}^{l o}} \sum_{n \in \Lambda_{i j}^{l o}} \sum_{m \in M_{n}^{l o}} \sum_{t \in K_{m}^{l o}} \exp \left(-\theta_{i}^{l o} u_{i j}^{l o n m t}+A_{j}^{l o}\right)
$$

Dividing Equation 35 by Equation 36 gives:

$$
T_{i j}^{l o}=G_{i}^{l o} \frac{\sum_{n \in \Lambda_{i j}^{l o}} \sum_{m \in M_{n}^{l o}} \sum_{t \in K_{m}^{l o}} \exp \left(-,{ }_{i}^{l o} u_{i j}^{l o n m t}+A_{j}^{l o}\right)}{\sum_{j \in D_{i}^{l o}} \sum_{n \in \Lambda_{i j}^{l o}} \sum_{m \in M_{n}^{l o}} \sum_{t \in K_{m}^{l o}} \exp \left(-,{ }_{i}^{l o} u_{i j}^{\text {lonmt }}+A_{j}^{l o}\right)}
$$


which is Equation (3) in the MSTEM.

Multiplying Equation 20 by $\theta_{i}^{l o}$ and use $G_{i}^{l o}=Q_{i}^{l o}-e_{i}^{l o}=\alpha^{l o} S_{i}^{l o}+E_{i}^{l o}$ gives:

$-\theta_{i}^{l o} \gamma_{i}^{l o}=\ln \left(\alpha^{l o} S_{i}^{l o}+E_{i}^{l o}\right)-S_{i}^{l o}-\theta_{i}^{l o} \eta_{i}^{l o}$

Substitute the right hand side of (37) in (35) gives:

$T_{i j}^{l o}=\frac{\left(\alpha^{l o} S_{i}^{l o}+E_{i}^{l o}\right) \sum_{n \in \Lambda_{i j}^{l o}} \sum_{m \in M_{n}^{l o}} \sum_{t \in K_{m}^{l o}} \exp \left(-\theta_{i}^{l o} u_{i j}^{l o n m t}+A_{j}^{l o}\right)}{\exp \left(S_{i}^{l o}+\theta_{i}^{l o} \eta_{i}^{l o}\right)}$

Summing Equation 38 over all $j \in D_{i}^{l o}$ and using constraint (9) of the ECP gives:

$\exp \left(S_{i}^{l o}+\theta_{i}^{l o} \eta_{i}^{l o}\right)=\sum_{j \in D_{i}^{l o}} \sum_{n \in \Lambda_{i j}^{l o}} \sum_{m \in M_{n}^{l o}} \sum_{t \in K_{m}^{l o}} \exp \left(-,{ }_{i}^{l o} u_{i j}^{l o n m t}+A_{j}^{l o} \quad\right)$

The optimality condition (26) implies that $\eta_{i}^{l o}=0$ whenever $S_{i}^{l o} \succ 0$ and, therefore, Equation 39 reduces to the following:

$S_{i}^{l o}=\ln \sum_{j \in D_{i}^{l o}} \sum_{n \in \Lambda_{i j}^{l o}} \sum_{m \in M_{n}^{l o}} \sum_{t \in K_{m}^{l o}} \exp \left(-,{ }_{i}^{l o} u_{i j}^{\text {lonmt }}+A_{j}^{l o} \quad\right) \succ 0$

Also, since $\eta_{i}^{l o} \geq 0$, the right hand side of Equation 39 is negative whenever $S_{i}^{l o}=0$ and, thus, accessibility is always given by the following:

$S_{i}^{l o}=\max \left\{0, \ln \sum_{j \in D_{i}^{l o}} \sum_{n \in \Lambda_{i j}^{l o}} \sum_{m \in M_{n}^{l o}} \sum_{t \in K_{m}^{l o}} \exp \left(-,{ }_{i}^{l o} u_{i j}^{\text {lonmt }}+A_{j}^{l o} \quad\right)\right\}$

which is Equation 1 in the MSTEM.

From Equation $31 \omega_{p}^{\text {lonmt }}=0$ if $h_{p}^{\text {lonmt }} \succ 0$, substituting in Equation 25 gives:

$\hat{C}_{p}^{\text {lonmt }}=\sum_{a \in A} \delta_{a p}^{\text {lonmt }} \hat{C}_{a}^{\text {lonmt }}\left(f_{a}^{\text {lonmt }}\right)=u_{i j}^{\text {lonmt }}$ whenever $h_{p}^{\text {lonmt }} \succ 0$.

Moreover, since $\omega_{p}^{\text {lonmt }} \geq 0$,

$\hat{C}_{p}^{\text {lonmt }}=u_{i j}^{\text {lonmt }}+\omega_{p}^{\text {lonmt }} \geq u_{i j}^{\text {lonmt }}$ whenever $h_{p}^{\text {lonmt }}=0$

That is: 


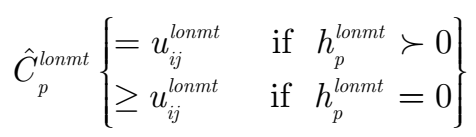

which is Equation 28 in the MSTEM.

Thus, the ECP and the diagonalized MSTEM $(\mathbf{C}=\hat{\mathbf{C}})$ are indeed equivalent. Existence, convexity, and uniqueness of the ECP problem can be followed as those of Safwat and Magnanti (1988).

\section{CONCLUSION}

Single class travel forecasting models assume that all travelers are similar in their travel-decision characteristics, such as their money-value of the time and their sensitivity to travel times in choosing their origin, destination, and mode of travel. To obtain more realistic models, travelers are often divided into classes, either by socioeconomic attributes (e.g., income level and car availability) or by the purpose (e.g., home-based-work, non-home-based-work, and home-based-shopping) of their travel, assuming that travel-decision characteristics are the same within each class but differ among classes.

The development of this concept of multiple classes increases the mathematical complexity of travel forecasting models.

The authors formulated the MSTEM as a VI problem, and proposed a relaxation (diagonalization) algorithm to solve it, where they formulated the diagonalized subproblem of the algorithm as an ECP that they proved to be equivalent to the diagonalized MSTEM.

The ECP can be solved by a multiclass logit distribution of trips algorithm. In order to enhance the computational aspect of the MSTEM, the authors formulated the ECP of the MSTEM as an FDUE problem using the concept of supernetwork representation. This FDUE problem can be easily solved by a simple greedy path-based algorithm for traffic assignment (Xie et al., 2018).

Comparison applications to real-world transportation network between the MSTEM with its original ECP using multiclass logit distribution of trips algorithm and its new ECP (as FDUE) using a simple greedy path-based algorithm for traffic assignment (Xie et al. 2018) are essential to prove the enhancement of the computational aspect of the MSTEM. 


\section{REFERENCES}

Beckman, M., McGuire, C. B., \& Winston, C. B. (1956). Studies in the economics of transportation. Yale University Press.

Boyce, D. E. (2014). Martin Beckmann in the 1950s: Contributions to regional science. In R. Funck \& W. Rothengatter (Eds.), Man, environment, space, and time - Economic interactions in four dimensions (pp. 15-35). Nomos. doi:10.5771/9783845260440_15

Boyce, D. E., \& Bar-Gera, H. (2004). Multiclass combined models for urban travel forecasting. Networks and Spatial Economics, 4(1), 115-124. doi:10.1023/B:NETS.0000015659.39216.83

Boyce, D. E., \& Nagurney, A. (2019). In Memoriam: Martin Beckmann (1924-2017). Transportation Science, 53(6), 1501-1799. doi:10.1287/trsc.2019.0942

Dafermos, S. C. (1982). Relaxation algorithm for the general asymmetric traffic equilibrium problem. Transportation Science, 16(2), 231-240. doi:10.1287/trsc.16.2.231

De Cea, J., Fernandez, J. E., Dekock, V., Soto, A., \& Friesz, T. L. (2003). ESTRAUS: A computer package for solving supply-demand equilibrium problem on multimodal urban transportation networks with multiple user classes [Paper presentation]. Annual Meeting of the Transportation Research Board, Washington, DC, United States.

Evans, S. P. (1976). Derivation and analysis of some models for combining trip distribution and assignment. Transportation Research, 10(1), 37-57. doi:10.1016/0041-1647(76)90100-3

Florian, M., \& Nguyen, S. (1978). A combined trip distribution mode split and trip assignment model. Transportation Research, 12(4), 241-246. doi:10.1016/0041-1647(78)90065-5

Florian, M., \& Spiess, H. (1982). The convergence of diagonalization algorithms for asymmetric network equilibrium problems. Transportation Research, 16, 447-483.

Hasan, M. K., \& Al-Gadhi, S. A. (1998). Application of simultaneous and sequential transportation network equilibrium models to Riyadh, Saudi Arabia. Transportation Research Record: Journal of the Transportation Research Board, 1645(1), 127-132. doi:10.3141/1645-16

Hasan, M. K., \& Al-Qaheri, H. (2013). Optimization and GIS-based intelligent decision support system for urban transportation systems analysis. International Journal of Transport and Vehicle Engineering, 7(3), 402-427.

Hasan, M. K., \& Dashti, H. M. (2007). A multiclass simultaneous transportation equilibrium model. [Springer, USA.]. Networks and Spatial Economics, 7(3), 197-211. doi:10.1007/s11067-006-9014-3

Safwat, K. N. A., \& Magnanti, T. L. (1988). A combined trip generation, trip distribution, modal split, and traffic assignment model. Transportation Science, 22(1), 14-30. doi:10.1287/trsc.22.1.14

Tatineni, M. R., Lupa, M. R., Englund, D. B., \& Boyce, D. E. (1994). Transportation policy analysis using a combined model of travel choice. Transportation Research Record: Journal of the Transportation Research Board, (1452), 10-17.

Xie, J., Nie, Y., \& Liu, X. (2018). A greedy path-based algorithm for traffic assignment. Transportation Research Record: Journal of the Transportation Research Board, 2672(48), 36-44. doi:10.1177/0361198118774236

Yao, J., Chen, A., Ryu, S., \& Shi, F. (2014). A general unconstrained optimization formulation for the combined distribution and assignment problem. Transportation Research Part B: Methodological, 59(C), 137-160. doi:10.1016/j.trb.2013.11.007

Zhao, Q., Turnquist, M. A., Dong, Z., \& He, X. (2018) Multiclass probit-based origin-destination estimation using multiple data types. Journal of Transportation Engineering, Part A: Systems, 144(6). 


\begin{abstract}
Mohamad K. Hasan (PhD) is a Professor of Operations and Supply Chain Management at Dept. of Information Systems and Operations Management, College of Business Administration Kuwait University, Kuwait. He received a B.S. degree in Mathematical Statistics from Alexandria University, Egypt, in 1978. He received M.S. degree in Mathematics in 1988 and Ph.D. degree in Transportation Systems Analysis and Planning in 1991 both from Texas A\&M University, College Station, Texas, USA. His research interests include: Logistics, Supply Chain Management, Network Optimization, Scheduling, Transportation Systems Analysis, Transportation Planning, Transportation Network Modeling, Freight Transportation Modeling, and Mathematical Programming. He presented his research at national and international conferences, including Transportation Research Board Annual Meetings and INFORMS. His articles in Transportation Research Record, Networks and Spatial Economics, Telecommunication Systems, Journal of Transportation Engineering, International Journal of Operations Research and Information Systems, International Journal of Computer Information Systems and Industrial Management Applications, Engineering (Scientific Research), Journal of Service Science \& Management(Scientific Research), Informatica (An International Journal of Computing and Informatics), Proceeding of ASCE (American Society of Civil Engineering) Transportation Congress, San Diego, California, U.S.A.), Proceeding of 1st CSCE (Canadian Society of Civil Engineering) Transportation Specialty Conference. Edmonton, Alberta, Canada, and Proceeding of 2nd CSCE (Canadian Society of Civil Engineering) Transportation Specialty Conference, Halifax, Nova Scotia, Canada.
\end{abstract}

Mohammad Saoud $(P h D)$ received the BS degree in computer engineering from Kuwait University, and the MBA and the Ph.D. degree in operations management from Purdue University. He is currently an Assistant Professor at the Department of Information Systems \& Operations Management at Kuwait University. His research interests include product architectures, sustainable operations, energy conservation, environmental regulation, transportation models, supply chain management and decision support systems.

Raed Al-Husain (PhD) received his Bachelor of Science in Electrical Engineering and his Master of Science in Operations Research Engineering, from the University of Southern California, Los Angeles, in 1999 and 2002 respectively. He received his Doctoral of Philosophy degree in Business Operations Management from the University of Houston, Texas, in 2005. Currently, he works as an Associate Professor in the Department of Information Systems and Operations Management, College of Business Administration in Kuwait University. Dr. Al-Husain Also held multiple administrative positions in the college, such as the director of the Office of Student Training and Alumni and the Information Technology Center, where he helped improve their operational capabilities in meeting both students and faculty needs. His research interests include logistics and supply chain management and design, production planning and scheduling, inventory management, quality management, and simulation. 